\title{
Einige Personennamen und Götternamen in schwedischen Ortsnamen
}

\author{
Von Sven Benson
}

Das Wissen über die religiösen Verhältnisse Schwedens im ersten Jahrtausend unserer Zeitrechnung ist aus den verschiedensten Quellen gewonnen worden. Die Archäologen finden in der Erde Gegenstände, die religiösen Symbolwert gehabt haben mögen. Archäologen und Kunsthistoriker interpretieren in Zusammenarbeit mit Religionshistorikern das Bildmaterial auf Steinen und losen Gegenständen. Die altisländische Literatur, besonders die Edda Snorris und die Poetische Edda, bieten ein grosses Material dar, das wahrscheinlich auch für den östlichen Norden relevant ist. Von Tacitus bis Adam von Bremen finden wir in aussernordischen Texten Auskünfte über kultische Verhältnisse im Norden. Etliche Runentexte enthalten kurze Phrasen, die sich auf religiöse Verhältnisse beziehen. Die Runensteine des elften Jahrhunderts sind besonders reich an christlichen und heidnischen Symbolen.

Ich habe nich die Absicht, hier einen Katalog über das ganze Material zu erstellen. Statt dessen will ich einen besonderen Quellentypus behandeln, nämlich die Ortsnamen. Es ist bekannt, dass die Ortsnamen vieles über die Religion vergangener Zeiten erzählen können. Nicht zuletzt können sie die Verhältnisse in den Jahrhunderten des grossen Religionswechsels beleuchten. Zunächst sei an einige wohlbekannte Tatsachen erinnert.

Eine grosse Menge unserer Ortsnamen können in sprachlicher Hinsicht als zusammengesetzte Wörter betrachtet werden. Thr erstes Glied ist oft ein Personenname, ihr zweites Glied kann z. B. die Bezeichnung einer Naturformation oder einer Siedlung sein. Religionsgeschichtlich interessant werden die Ortsnamen, wenn das erste Glied ein Göttername ist oder wenn das zweite Glied eine Lokalität bezeichnet, wo möglicherweise kultische Tätigkeiten ausgeübt wurden. Beide Bedingungen werden von dem dänischen Stadtnamen Odense erfüllt. Adam von Bremen verwendet die Form Odansue. Das erste Glied ist der Göttername Oden, das zweite das Substantivum vi, das ein heidnisches Heiligtum bezeichnete. 
Die Götternamen, die wir in schwedischen Ortsnamen finden, sind vor allem Tor, Oden, Frö, Fröja, Njärd und Ull. Substantivische Namenelemente, die auf Kultplätze schliessen lassen, sind u.a. vi, harg land, lund, tuna und aker. Von jetzt verschwundenen Substantiven kann das Wort *al erwähnt werden, das 'Tempel' bedeutete. Die meisten der genannten Namenelemente, z. B. harg, land, lund, tuna und aker sind auch in profanen Zusammenhängen verwendet und mit nicht-theophoren Bestimmungswörtern verbunden worden.

Unter schwedischen Ortsnamen, die allgemein für theophore Namen gehalten werden, kann man Torshälla (ält. Thorsharghi u.d.), Torslunda, Torstuna, Torsåker; Onsala, Onslunda, Odensala (ält. Othinsharg), Odensvi, Odensåker; Fröseke, Fröslunda, Frösvi; Frölunda, Frötuna, Frövi, Fryele, Friel; Närlunda, Närtuna; Ulleråker, Ultuna erwähnen.

Neben den von heidnischer Religion geprägten Ortsnamen lag zu der Zeit, wo das Christentum in Schweden eingeführt wurde, ein grosser profaner Ortsnamenschatz vor. In vielen dieser Namen bildeten Personennamen das erste Glied. Besonders gewöhnlich waren Personennamen in Ortsnamen, die mit löv, lev zusammengesetzt waren. Die in diesen Namen vorliegenden Personennamen hatten einen altertümlichen Charakter. Der schonische Ortsname Görlöv hat vermutlich den Personennamen Götar (aus ält. Gautaharjar), einen ursprünglich zweigliedrigen Namen, enthalten. In Arlöv liegt der eingliedrige Personenname Ari vor. Ortsnamen, die auf $h o ̈ g$ (oder daraus entwickeltes $i e$ ) enden, enthalten auch altertümliche Personennamen. In Hammenhög und (Västra und Östra) Vemmenhög liegen die Männernamen Hamund und Wemund vor. In Arrie (1120 Arrøgum) hat man den oben genannten Namen Ari sehen wollen.

$\mathrm{Zu}$ dieser Zeit waren jedoch andere einheimische einglied rige Namen wie Karl, Sven, Knut, Katil und Gisle und vormals zweigliedrige Namen wie Erik, Gunnar, Harald und Olof (Olav) häufiger. Diese Namen kommen auch in isländischen Sagas oft vor, deren Handlung im früheren Mittelalter und in verschieden Teilen des Nordens spielte.

Das Christentum brachte neue Männernamen wie Andreas, Benedictus, Johannes, Laurentius, Mattias, Paulus und Petrus mit sich. Diese Namen liegen in heutigen Ortsnamen in der Regel als Anders, Bengt, Jon, Lars, Matt(i)s, Pål, Påvel und Per vor.

Die Untersuchung, mit der ich beschäftigt bin, gilt u.a. der Konkurrenz zwischen alten, nordischen Personennamen und neueingeführten, christlichen Namen bei der Ortsnamenbildung in Schweden. Der Hauptteil unserer Gemeindenamen, die Mehrzahl unserer Dorfnamen und 
viele unserer Hofnamen wurden im Mittelalter oder früher gebildet. Im Laufe der Zeit erlitten viele Ortsnamen Denotationsklassenänderungen.

Bei der Durchsicht einiger reichsumfassender schwedischer Ortsnamenverzeichnisse späterer Zeiten fand ich, dass die genannten nordischen und christlichen Namen immer noch eine quantitativ führende Stellung in dem schwedischen Ortsnamenschatz einnehmen. Von den alten Götternamen ist nur Tor in späteren Zeiten produktiv gewesen, doch nicht als Göttername, sondern als Personenname.

Der häufige Gebrauch des Namens Olof, Olav (und daraus entwickelter Namenformen) bei mittelalterlicher nordischer Ortsnamenbildung hängt mit dem gewaltsamen Tode und der Heiligsprechung des norwegischen Königs Olav Haraldsson zusammen. Die eigenartige Situation entstand, dass ein alter, nordischer Name in besonders hohem Grad mit dem neuen, christlichen Kultus verbunden wurde. Demselben kultischen Gebrauch unterlagen die Heligennamen Erik und Knut nicht, obwohl auch der schwedische Heiligenname Erik und der dänische Heiligenname Knud von Königen getragen wurden. Der Name Olof, Olav und die daraus entwickelten Namenformen erfordern eine Sonderuntersuchung und werden hier nicht behandelt.

Die Personennamen, die hier als Ortsnamenelemente untersucht werden, sind die einheimischen, nordischen Namen Sven, Karl, Erik und Knut und die eingeführten, christlichen Namen Per, Anders, Jon und Bengt. Lars, Matt( $i) s$ und Pål treten spärlicher als die anderen christlichen Personennamen in Ortsnamen auf. Matt $(i) s$ kommt vor allem in gotländischen Ortsnamen vor und ist in den übrigen Teilen Schwedens als Ortsnamenelement sehr spärlich belegt.

Die Ortsnamen, die irgendeinen von den erwähnten Männernamen enthalten, habe ich statistisch in geographischer, typologischer und funktioneller Hinsicht untersucht.

Für meine Untersuchung benötigte ich eine Quelle, die die Ortsnamen in allen Teilen Schwedens einheitlich vorlegt. Ich entschied mich für C. M. Rosenberg, Geografiskt-statistiskt handlexikon öfver Sverige (Rosenberg 1982 (1881-83)). Rosenberg benutzt vor allem die Grundsteuerrollen des Jahres 1878 als Primärmaterial. Das bedeutet, dass die Namen aller Dörfer und auch selbständiger Höfe ausserhalb der Dorfschaft im Prinzip erwähnt werden. Dazu kommt eine nicht geringe Menge Namen, die Naturformationen und administrative Einheiten verschiedener Art bezeichnen. Das Lexikon Rosenbergs enthält etwa 70000 Namen. Ich schätze, dass etwa 62000 davon Siedlungen bezeichnen. Der erste Schritt meiner Untersuchung ist, die Verteilung 
der Ortsnamen während des Jahres 1878 festzustellen, die die untersuchten Personennamen enthalten.

Bei der Untersuchung der Verteilung wird folgende Methode verwendet. Zuerst wird berechnet, wieviele Siedlungsnamen in jedem Regierungsbezirk ("län") vorkommen. Die gefundenen Werte werden in Prozentzahlen umgewandelt. Ich habe danach untersucht, wie hoch der Prozentanteil der Ortsnamen mit den betreffenden Personennamen in jedem einzelnen Regierungsbezirk ist. Die beiden Prozentzahlen jedes einzelnen Bezirks werden miteinander verglichen. Von allen Ortsnamen, die den Männernamen Karl enthalten, werden $7,02 \%$ in dem Regierungsbezirk Södermanlands län angetroffen. In diesem Regierungsbezirk liegen 7,15\% aller Ortsnamen Rosenbergs vor. Wenn die erste Zahl durch die zweite dividiert wird, bekommen wir die Quote 0,98. Diese Zahl liegt der Nullhypothese sehr nahe, die besagt, dass die Quote jedes einzelnen Regierungsbezirks 1,00 sein soll. Wenn die Quote eines Regierungsbezirks grösser als 1,3 ist, betrachte ich die relative Frequenz oder Dichte des Namenelements in diesem Bezirk als hoch. Wird die Quote höher als 2,0, betrachte ich die relative Frequenz als sehr hoch. Eine Quote, die 0,8 oder niedriger ist, zeugt von niedriger relativer Frekvenz. Die niedrigste Quote, 0,0, entsteht, wenn das Namenelement in einem Regierungsbezirk völlig fehlt.

Wir werden bemerken, dass hohe Werte oft in Regierungsbezirken vorkommen, die aneinander grenzen und grössere, zusammenhängende Gebiete bilden. Für alle untersuchten Namen und Bezirke sind die absoluten Zahlen so gering, dass unerwartet hohe oder niedrige Werte für einzelne Bezirke entstehen können. Man muss die relative Frequenz grösserer Gebiete beachten (vgl. Benson 1989, 9 ff.).

Der Name Sven kommt bei Rosenberg als Namenelement in 136 Ortsnamen vor. Diese Namen sind in den südwestlichsten Teilen Schwedens am zahlreichsten belegt. Die Bezirke Blekinge, Kristianstads, Malmöhus, Hallands, Göteborgs och Bohus, Älvsborgs, Skaraborgs, Jönköpings und Värmlands län bilden ein zusammenhängendes Gebiet, wo 102 Ortsnamen vorkommen, die den Personennamen Sven enthalten. Das bedeutet 75,0\% aller solcher Namen. In diesem Gebiet begegnen $42,5 \%$ aller Ortsnamen. Die Quote beträgt 1,8. In dem angrenzenden Bezirk Kronobergs län ist die Quote 1,0. Besonders hoch $(2,6)$ ist die Quote in Schonen (Malmöhus und Kristianstads län), und auch in Värmlands län erreicht sie immerhin einen Wert von 2,1. In den übrigen Teilen Schwedens ist die relative Frequenz dagegen gering. Die Quote des ganzen Restgebiets ist 0,4 .

Dass der Personenname Sven zu allen Zeiten im südlichen und west- 
lichen Schweden am häufigsten gewesen ist, hat Roland Otterbjörk in Svenska förnamn (Otterbjörk 1964) hervorgehoben. Seine Beobachtung wird, was das Mittelalter betrifft, von Staffan Hellberg in Språk och tradition (Hellberg 1983, 78-85) bestätigt.

Die Ortsnamengruppe im untersuchten Material, die den Personennamen Karl enthält, ist schwierig abzugrenzen. Schon im Mittelalter konnte - $l$ - in der Verbindung - rls- fallen, wobei sich z. B. der Name Karlstorp zu Karstorp entwickelte. In älteren Urkunden wechseln oft Karlstorp und Karstorp als Bezeichnungen derselben Lokalität. Der Name Karlsröd entwickelte sich im nordwestlichen Götaland über Karsröd zu Karseröd ebenso wie Svensrud zu Svenserud und Persröd zu Perseröd. Ausserdem konnte - $r$ - zuweilen in der Verbindung - rlsausfallen, wobei ein Erstbestandteil Kals- entstand.

Anderseits kommen in Rosenbergs Material etliche zusammengesetzte Namen vor, deren Erstbestandteil Karse- oder Karsa- war und die offenbar niemals den Männernamen Karl enthalten haben oder damit assoziiert worden sind.

Ich habe in meine Statistik die Namen aufgenommen, die nun Karl-, Karls-, Kars- und Kals- enthalten, und daneben auch Karseröd. Einige der Ortsnamen haben den Männernamen Karl ursprünglich nicht enthalten, sondern sind sekundär damit assoziiert worden. In den meisten Fällen mit Kars- und Kals- gibt es jedoch ältere Schriftformen mit Karls-.

In der Gruppe, die in dieser Weise abgegrenzt worden ist, liegen 242 Ortsnamen vor. Ich bin mir dessen bewusst, dass einzelne dieser Namen vielleicht niemals von den Namengebern oder Namengebrauchern mit dem Männernamen Karl verknüpft worden sind.

Die Ortsnamen, die zu dieser Gruppe gehören, zeigen eine hohe relative Frequenz in Östergötlands, Jönköpings, Skaraborgs und Örebro län, d.h. in den Regierungsbezirken um den Vättersee, und nördlich davon in Kopparbergs und Gävleborgs län. Für dieses Gebiet erhalten wir die Quote 1,7 als Ausdruck der relativen Frequenz des Ortsnamenelements Karl.

Der Personenname Erik hat in Ortsnamen die beiden Kompositionsformen Eriks- und Ers-. Die letztgenannte Form ist als Kompositionsform der verkürzten Variante Erk zu betrachten. Eriks- und Erskönnen in gewissen Gegenden, doch nicht im südlichsten Schweden, miteinander wechseln. 115 Ortsnamen enthalten Erik in einer dieser beiden Varianten. Das Kartenbild dazu ist diffus. Hohe relative Frequenz begegnet in Östergötlands, Älvsborgs, Skaraborgs, Kopparbergs und Västernorrlands län, sehr hohe Frequenz in Västerbottens län. 
Der Männername Knut liegt in weniger Ortsnamen als die oben behandelten Männernamen vor, insgesamt 79 Ortsnamen. Auch hier ist das Kartenbild diffus. Hohe relative Frequenz kommt in Kristianstads, Hallands, Jönköpings, Södermanlands und Örebro län vor. Sehr hohe relative Frequenz wird in Kopparbergs län erreicht. In Gävleborgs und Jämtlands län finden wir dagegen nur je einen Beleg. Sonst fehlt Knut ganz als Ortsnamenelement in dem norrländischen Material Rosenbergs. Allgemein gesehen hat Knut als Ortsnamenelement eine südlichere Orientierung als Erik.

Das am häufigsten belegte Ortsnamenelement der christlichen Gruppe ist Per. 101 Ortsnamen mit diesem Namenelement liegen vor. Das Kartenbild ist unklar. Sehr hohe relative Frequenz wird in Kristianstads, Hallands, Jönköpings, Värmlands und Kopparbergs län erreicht, hohe relative Frequenz in Blekinge, Kalmar, Skaraborgs, Västmanlands und Norrbottens län.

Anders ist in Ortsnamen spärlicher belegt. Nur 57 Siedlungsnamen, die Anders als Erstbestandteil enthalten, liegen bei Rosenberg vor. Einige der Ortsnamen dürften ursprünglich mit dem heidnischen Namen Andor (< Arnpor) gebildet worden sein. Die Belege sind über ganz Schweden verteilt. Die statistische Untersuchung zeigt sehr hohe Frequenz in Uppsala, Gotlands, Skaraborgs, Kopparbergs und Västerbottens län, hohe relative Frequenz in Hallands, Jönköpings und Västmanlands län.

Der Name Jon stellt als Ortsnamenelement ein eindeutigeres Bild dar. Von den 73 Ortsnamen ist der Hauptteil in den südlichen und westlichen Teilen Schwedens konzentriert. In einem zusammenhängenden Gebiet, das aus Kristianstads, Hallands, Älvsborgs, Skaraborgs und Värmlands län besteht, finden wir $63,0 \%$ aller Siedlungsnamen, die den Männernamen Jon enthalten. In diesem Gebiet finden wir nur $25,2 \%$ aller Siedlungsnamen Schwedens, und die Quote beträgt 2,5.

Bengt, schliesslich, ist das am spärlichsten belegte der analysierten Ortsnamenelemente. Nur 42 Namen sind belegt, und diese sind hauptsächlich in einem Gebiet Mittelschwedens konzentriert. In Skaraborgs, Värmlands, Örebro, Västmanlands, Kopparbergs und Uppsala län liegen $73,5 \%$ aller der Namen vor, die Bengt enthalten. Da nur 22,6\% aller Siedlungs-Namen Schwedens in diesem Gebiet vorkommen, ist die Quote, die die relative Frequenz ausdrückt, nicht geringer als 3,3. Diese Quote ist die höchste, die ich in einem grösseren, zusammenhängenden Gebiet gefunden habe.

Was die geographische Verbreitung bzw. Konzentration betrifft, gibt es keine prinzipiellen Unterschiede zwischen den gewöhnlichsten ein- 
Sven als

Ortsnamenelement

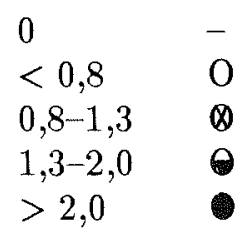

Rosenberg $n=136$

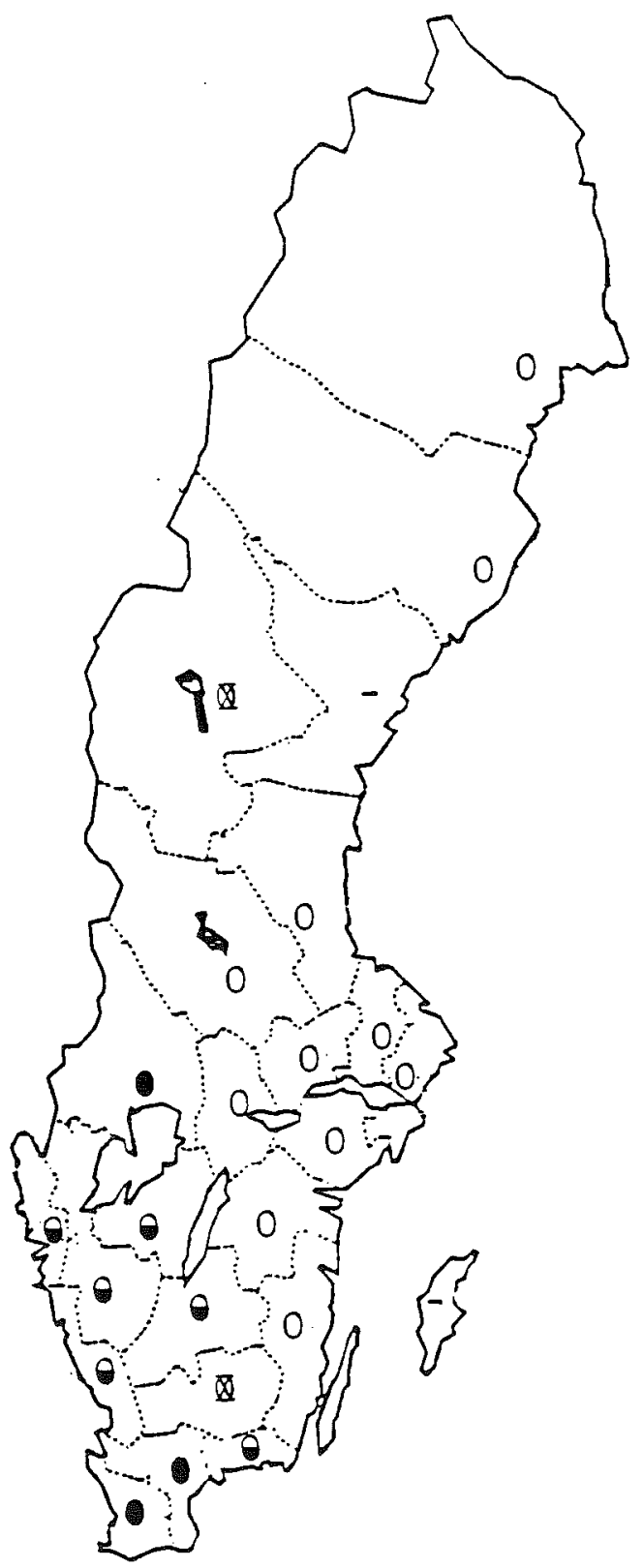

Karte 1. Relative Frequenz des Namens Sven als Ortsnamenelement 
Karl als

Ortsnamenelement

$<0,8 \quad \mathrm{O}$

$0,8-1,3 \bigotimes$

$1,3-2,0 \quad \odot$

Rosenberg $n=242$

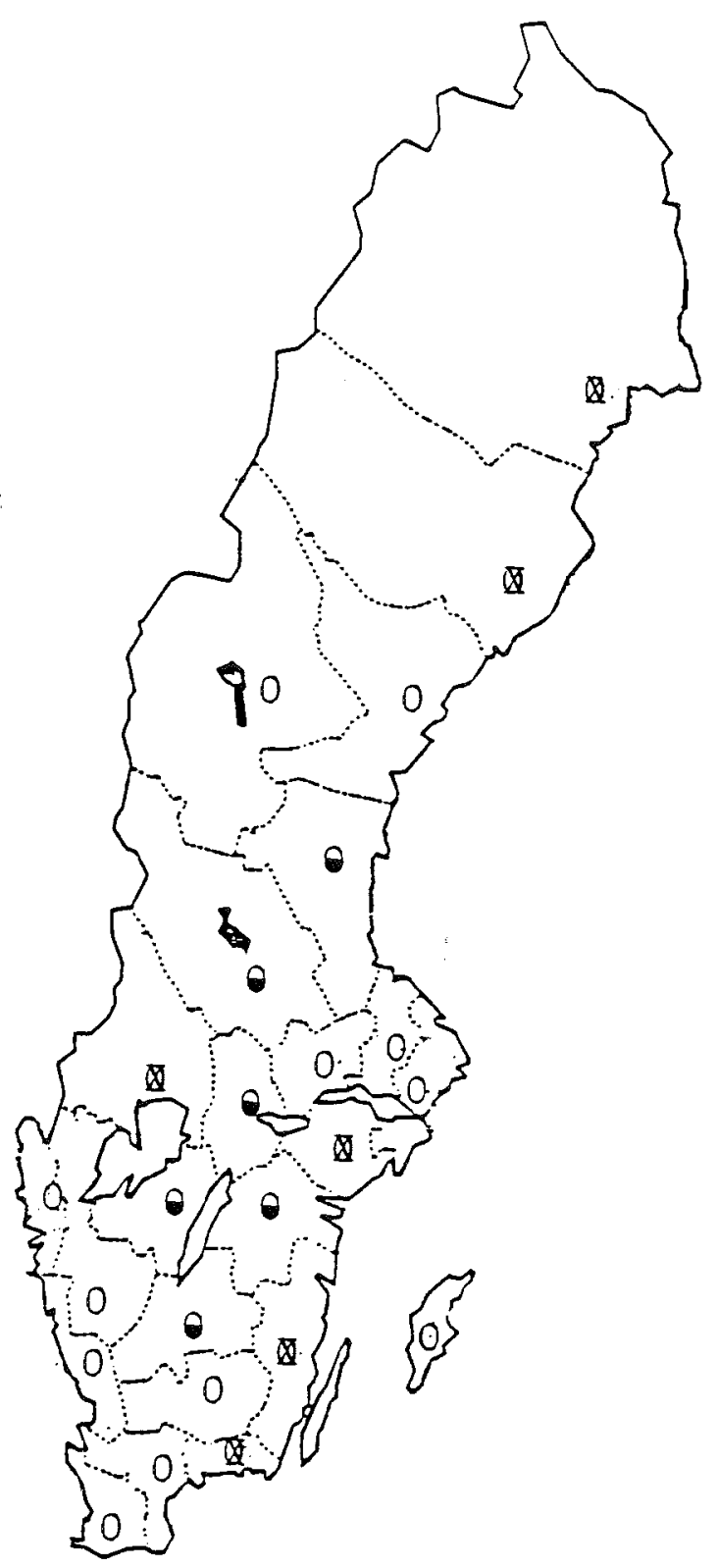

Karte 2. Relative Frequenz des Namens Karl als Ortsnamenelement 
Erik als

Ortsnamenelement

0

$<0,8 \quad 0$

$0,8-1,3 \quad \otimes$

$1,3-2,0$

$>2,0$

Rosenberg $n=115$

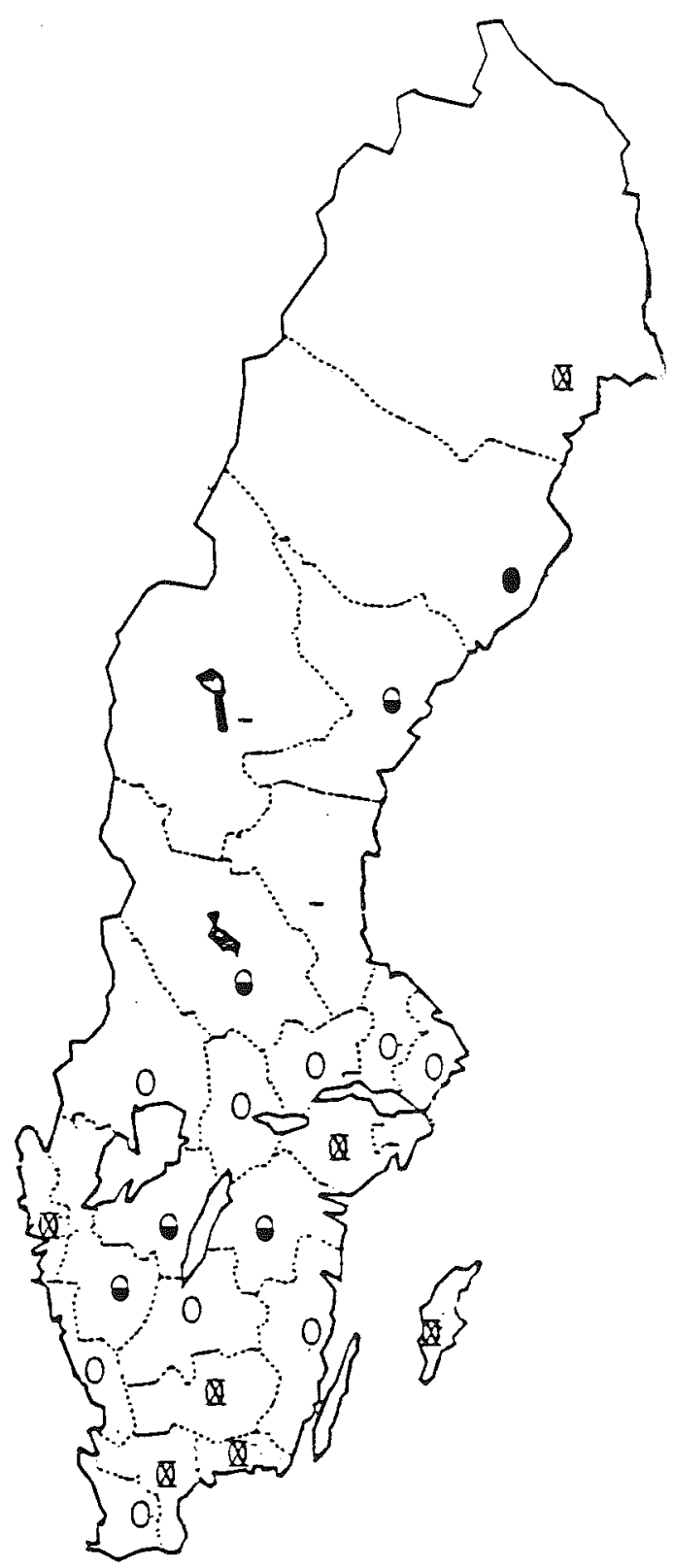

Karte 3. Relative Frequenz des Namens Erik als Ortsnamenelement 
Knut als

Ortsnamenelement

0

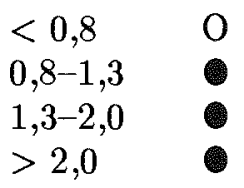

Rosenberg $n=79$

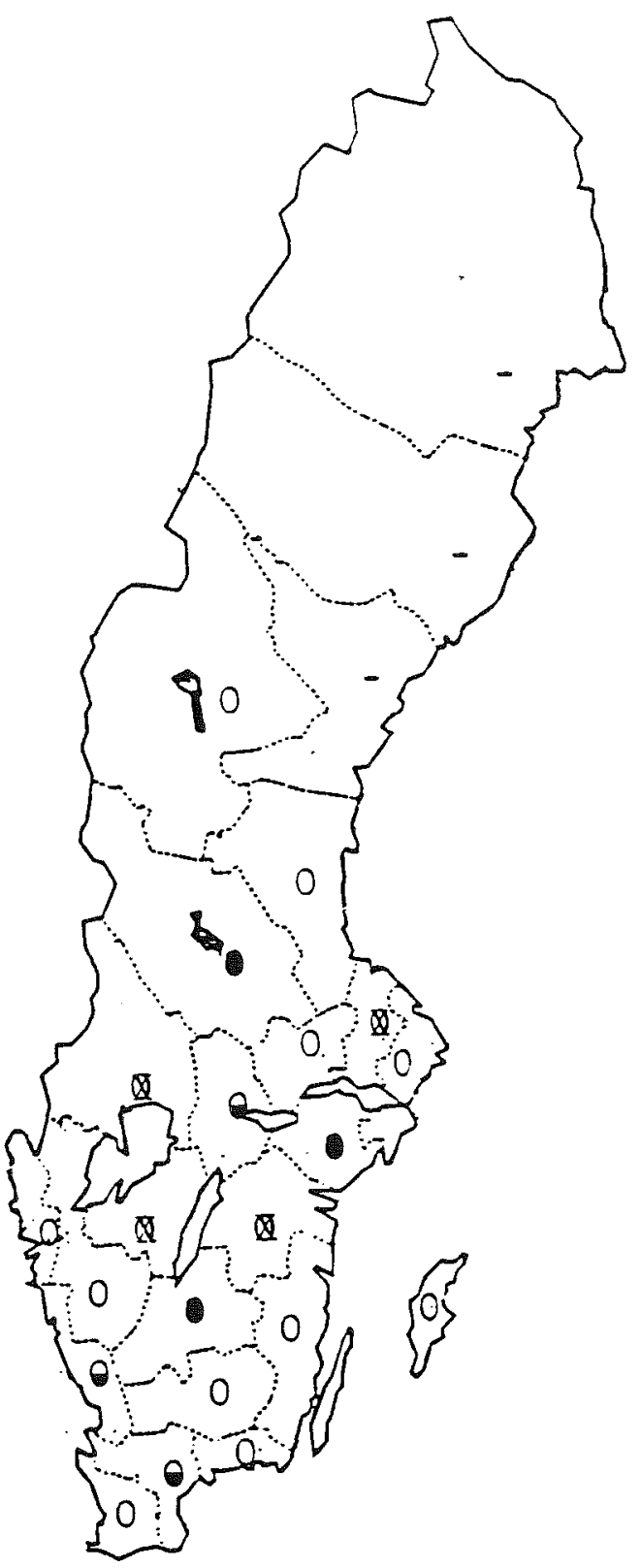

Karte 4. Relative Frequenz des Namens Knut als Ortsnamenelement 
Per als

Ortsnamenelement

0

$<0,8 \quad 0$

$0,8-1,3 \bigotimes$

$1,3-2,0 \quad 0$

$>2,0$

Rosenberg $n=101$

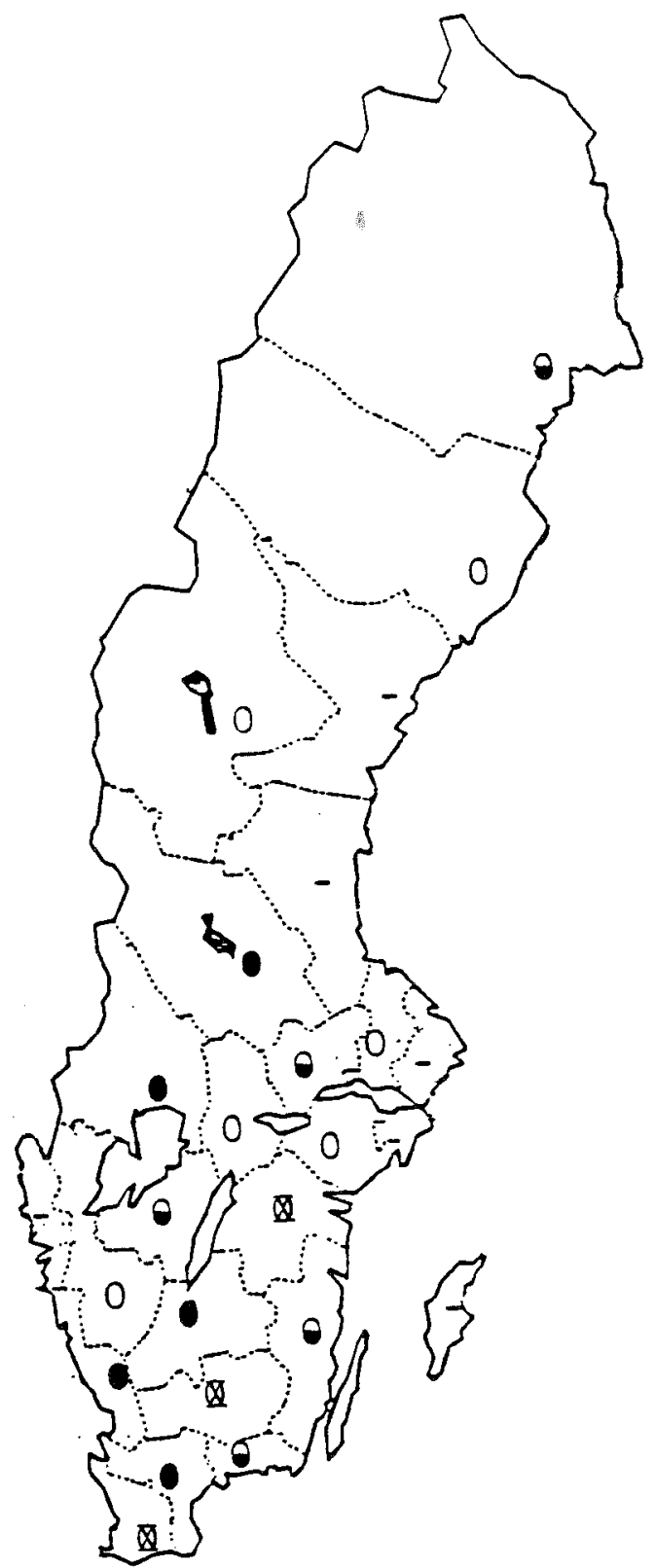

Karte 5. Relative Frequenz des Namens Per als Ortsnamenelement 
Anders als

Ortsnamenelement

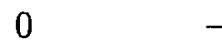

$<0,8 \quad 0$

$0,8-1,3 \bigotimes$

$1,3-2,0$

$>2,0$

Rosenberg $n=57$

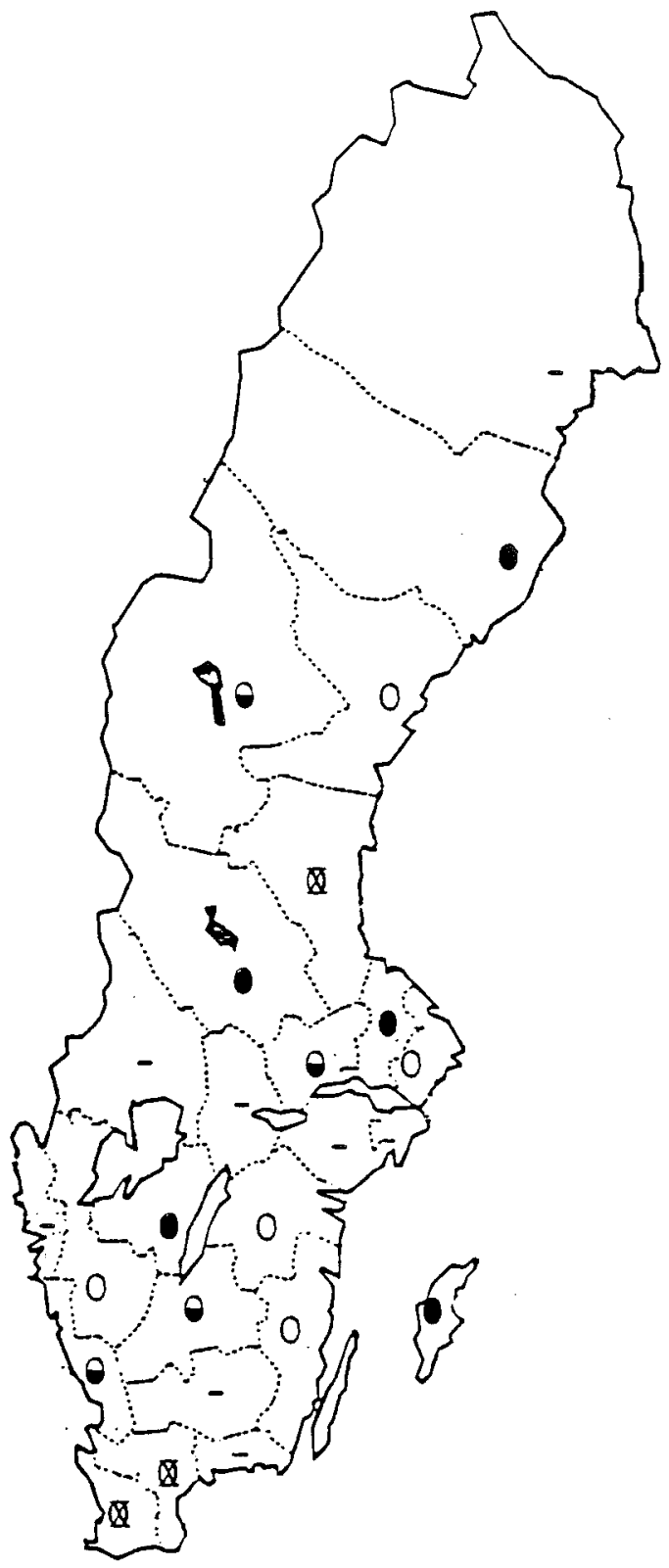

Karte 6. Relative Frequenz des Namens Anders als Ortsnamenelement 


\section{Jon als}

Ortsnamenelement

0

$<0,8 \quad$ O

$0,8-1,3 \bigotimes$

$1,3-2,0 \quad \ominus$

$>2,0$

Rosenberg $n=73$

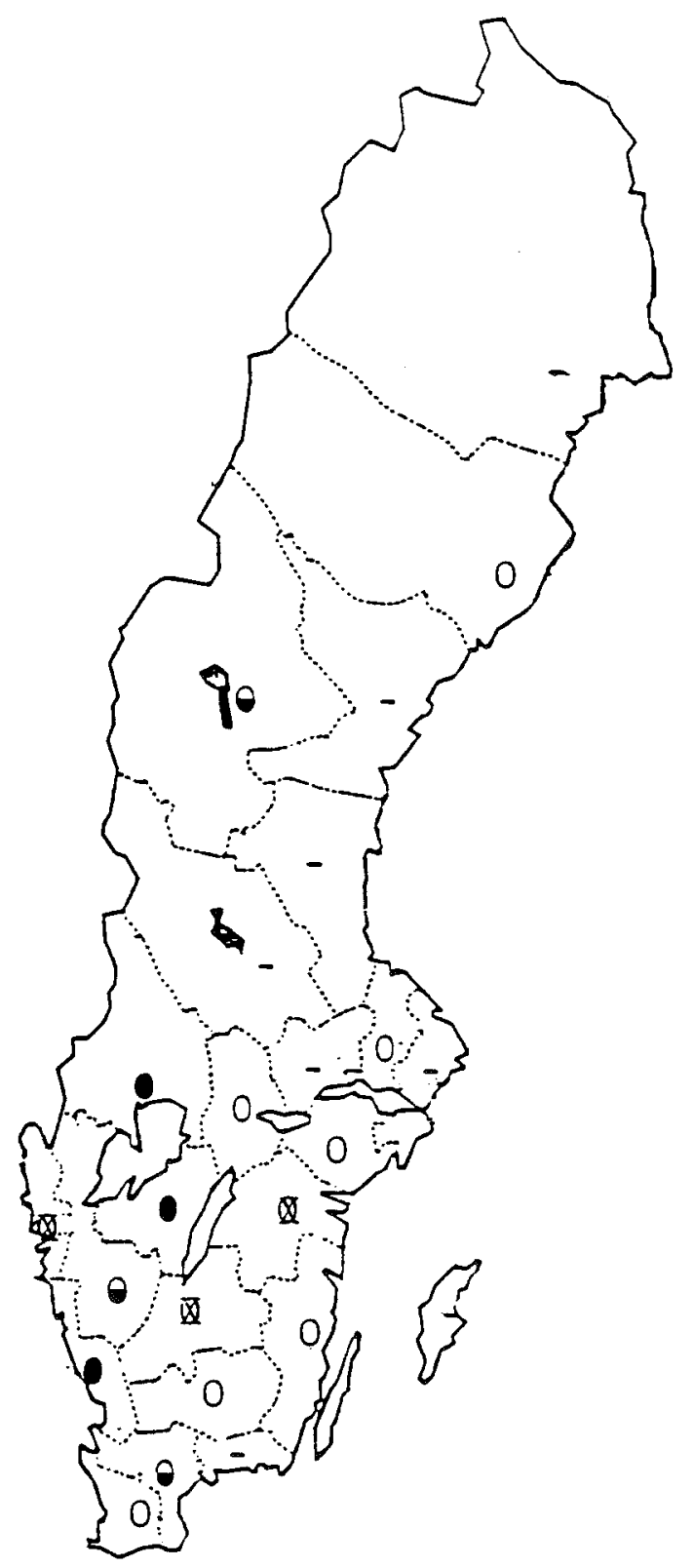

Karte 7. Relative Frequenz des Namens Jon als Ortsnamenelement 
Bengt als

Ortsnamenelement

0

$<0,8$

$0,8-1,3$

$1,3-2,0$

$>2,0$

Rosenberg $n=42$

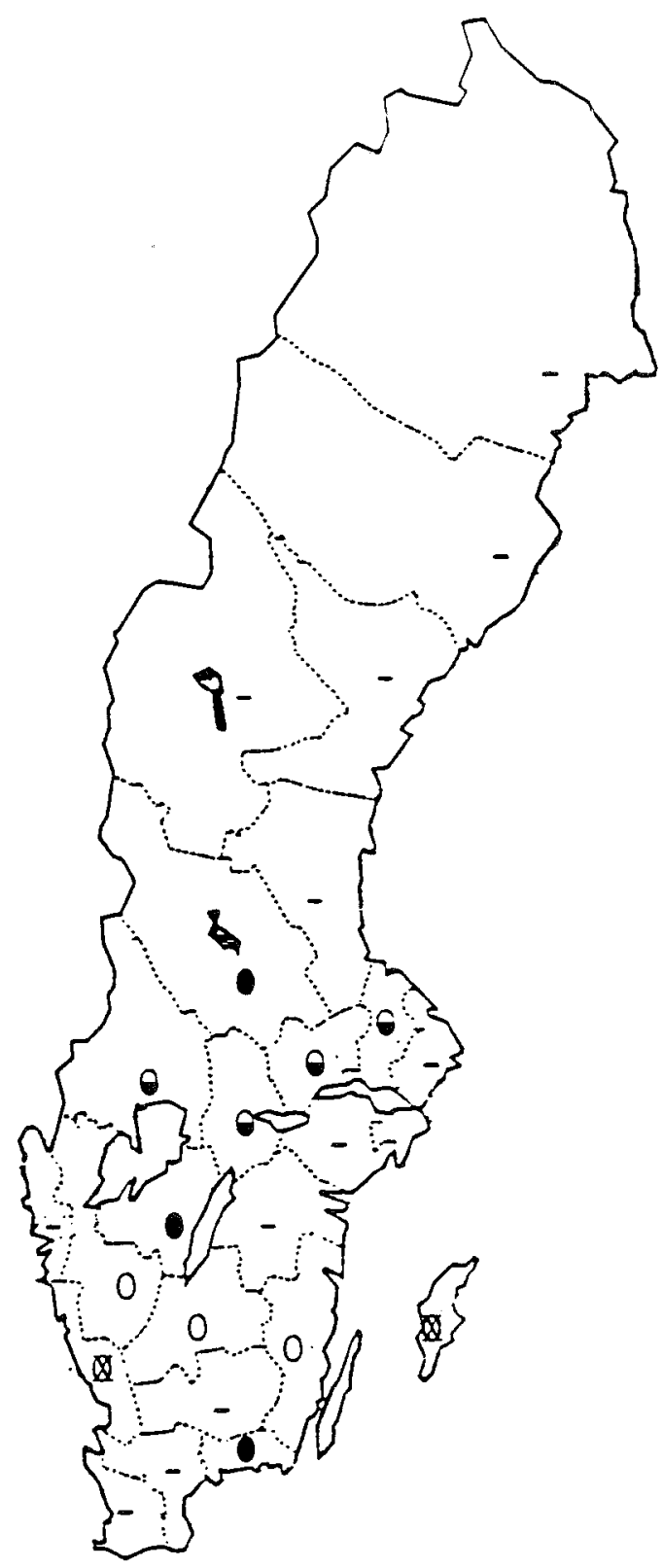

Karte 8. Relative Frequenz des Namens Bengt als Ortsnamenelement 
heimischen und den gewöhnlichsten eingeführen Namen als Ortsnamenelemente. Der einheimische Name Sven und der eingeführte Name Jon haben beide südliche und westliche Orientierung. Der einheimische Name Karl und der eingeführte Name Bengt sind beide zentralschwedisch orientiert, wenn auch mit verschiedenen Kartenbildern. Die nordischen Namen Erik und Knut haben ebenso wie die neueingeführten Namen Anders und Per eine Verbreitung ohne geographischen Schwerpunkt. In Norrland sind der eingeführte Name Anders und der nordische Name Erik üblicher, als man hätte erwarten können.

In absoluten Zahlen erreicht die einheimische Gruppe eine doppelt so grosse Verwendung wie die eingeführte. Nach meiner Rechnung stehen die beiden Namentypen in einem Verhältnis von 572:273.

Die vier hochfrequenten einheimischen Namen, die hier behandelt worden sind, werden nicht mit Grundwörtern des Typs zusammengesetzt, der mit heidnischen Götternamen verbunden wurde oder sonst von heidnischen Kulten zeugen kann. Dasselbe gilt natürlich auch für die christlichen Personennamen.

In den Jahrhunderten, wo das Christentum in Schweden an Boden gewann, wurde torp (thorp) ein sehr häufiges Grundwort. Der Gebrauch dieses neuen Grundworts kam vom Süden, es breitete sich über Götaland und Svealand nach Norden aus aber kam am Dalelf zum Stehen. Dieses Grundwort zeugt von einer starken, inneren Kolonisation während und nach der Wikingerzeit. Es dürfte in den meisten Fällen 'Ansiedlung' bedeutet haben, selbst wenn die Bedeutung allmählich an Prägnanz verlor. Neue, christliche Personennamen wurden also gerade zu der Zeit gebräuchlich, als das Grundwort thorp stark produktiv wurde. Ein tieferer Zusammenhang dürfte jedoch nicht vorliegen.

Die acht Vornamen werden in Rosenbergs Material in wechselndem Umfang mit torp verbunden. Von allen Ortsnamen, die Sven enthalten, sind $46 \%$ Zusammensetzungen mit torp. Die entsprechenden Prozentzahlen betragen für $\operatorname{Karl} 26$, für Erik 25, für Knut 37, für Per 47, für Anders 32, für Jon 41 und für Bengt 36. Karl und Erik werden proportional gesehen nicht so oft wie die anderen Namen mit torp verbunden. Dagegen werden diese beiden Namen öfters mit den mehr oder weniger nobilitierenden Grundwörtern berg, borg, dal, fors, holm, lund, näs und vik verbunden.

Meine Untersuchungen berufen sich wie gesagt auf ein Material vom Jahre 1878. Alle Ortsnamen, die heutzutage einen von den behandelten Personennamen enthalten, sind natürlich nicht im Mittelalter gegeben worden. Besonders Namen von Höfen und kleineren Lokalitäten 
sind späteren Datums. Das Material, das ich hier vorgelegt habe, stellt das Resultat einer Namengebung und eines Namenswandels dar, eines Prozesses, der mehr als tausend Jahre gedauert hat. Eine vorläufige Untersuchung der Ortsnamen i Skaraborgs län, die mit einem von den Namen Anders, Bengt, Karl, Erik oder Jon zusammengesetzt sind, zeigt, dass ungefähr die Hälfte der Siedlungsnamen Rosenbergs, die einen von diesen Namen enthalten, im 16. Jahrhundert oder früher zum ersten Mal belegt sind. Ich habe keinen grösseren Unterschied zwischen den verschiedenen Namen bemerkt.

Die heutige Frequenzverteilung von Ortsnamen, die Personennamen enthalten, dürfte eine mittelalterliche, örtlich wechselnde Popularität der Personennamen widerspiegeln. Die untersuchten Personennamen wurden von Leuten getragen, von und nach welchen die Ortsnamen gegeben wurden. Der häufige Gebrauch sowohl der alten, nordischen als auch der neuen, christlichen Personennamen bei der Bildung von Zusammensetzungen mit thorp deutet darauf hin, dass die zwei $\mathrm{Na}$ mengruppen schon früh namensoziologisch gleichgestellt wurden. Karl und Erik, die heute zu unseren allerhäufigsten Männernamen gehören, haben vielleicht eine besondere Rolle bei späterer Namengebung gespielt.

Für die einheimischen Personennamen lassen sich die Gründe der örtlichen Variation schwer feststellen. Was die christlichen Namen betrifft, muss man davon ausgehen, dass die Kirche den lokalen und regionalen Namengebrauch beeinflusst hat.

Von den etwa 850 Siedlungsnamen, die irgendeinen von den acht untersuchten Personennamen enthalten, bilden nur knapp $2 \% \mathrm{Ge}-$ meindenamen. Insgesamt handelt es sich um etwa 15 Gemeindenamen. Dazu kommen die in neuerer Zeit gegebenen Städtenamen Karlshamn, Karlskrona, Karlskoga und Karlstad. Dagegen sind von den etwa 45 Siedlungsnamen, die eine Variante des Götternamens Oden enthalten, nicht weniger als 7 , d.h. etwa $15 \%$, Gemeindenamen. Den Götternamen Tor hat man in mindestens 12 Gemeindenamen sehen wollen. In ungefähr ebenso vielen Gemeindenamen hat man die Götternamen Frö und Fröja sehen wollen. Heidnische Götternamen haben bei der Bildung von Gemeindenamen eine grössere Rolle als die untersuchten Männernamen gespielt. Die Entstehung der Gemeinden ist noch in vieler Hinsicht in Dunkel gehüllt, aber die Gemeindenamen haben offenbar nicht selten ihren Grund in heidnischen Vorstellungen und Sitten und im älteren, heidnischen Namenschatz. 


\section{Literat urverzeichnis}

Benson, S. 1989. Några personnamn i svenska ortnamn. En namnstrukturell studie. Studia Onomastica. Stockholm.

Hellberg, S. 1983. Namnet Sveinn i äldsta tid. Språk och tradition. Uppsala. Otterbjörk, R. 1964. Svenska förnamn, Stockholm.

Rosenberg, C. M. 1982 (1881-83). Geografiskt-statistiskt handlexikon öfver Sverige. [Hrsg. von] Landsarkivet i Göteborg och Genealogisk ungdom. Göteborg.

Bei der Durchsicht des Manuskripts war Fil. Dr. Peter Lafrenz behilfich. Ich danke ihm herzlichst. 